\title{
Combined exposure to dog and indoor pollution: incident asthma in a high-risk
} birth cohort

\author{
C. Carlsten*, M. Brauer*, H. Dimich-Ward*, A. Dybuncio*, \\ A.B. Becker ${ }^{\#}$ and M. Chan-Yeung*
}

ABSTRACT: The impact of single exposures on asthma development is better understood than the effect of multiple exposures. The objective of the present study was to evaluate the effect of combined early exposure to dog allergen (Can-f1) plus indoor nitrogen dioxide $\left(\mathrm{NO}_{2}\right)$ or environmental tobacco smoke (ETS) on asthma and bronchial hyperreactivity (BHR) in a high-risk birth cohort. We also aimed to assess atopy's impact on the effects of these exposures.

Peri-birth ETS exposure was measured using cord blood cotinine (CCot). During year 1, atopy, $\mathrm{NO}_{2}$, Can-f1, and urinary cotinine (UCot) were measured. At 7 yrs of age, 380 children were assessed for asthma and BHR. Exposure effects were determined using stepwise multiple linear regression.

Co-exposure to elevated Can-f1 and $\mathrm{NO}_{2}$, or Can-f1 and ETS (CCot), increased risk for asthma, relative to having neither such exposure (OR 4.8 (95\% Cl 1.1-21.5) and 2.7 (1.1-7.1), respectively); similar risks resulted when substituting dog ownership for allergen. Atopy increased asthma and BHR risk associated with several exposures; notably, atopy with elevated UCot, relative to atopy without such exposure, increased risk of BHR (OR 3.1 (95\% CI 1.1-8.6)).

In a high-risk birth cohort, early co-exposure to Can-f1 and $\mathrm{NO}_{2}$ or ETS increased the risk of incident asthma. Atopy increased the risk of asthma and BHR associated with ETS.

KEYWORDS: Asthma, atopy, dog, environmental tobacco smoke, nitrogen dioxide

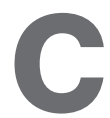

hildhood asthma is common and childhood allergic diseases have increased significantly in recent decades [1]. While it appears that exposure to allergens often promotes allergen sensitisation [2], the role of allergen exposure in asthma initiation is still controversial $[2,3]$, and there is evidence that environmental pollutants and respiratory infections are influential in the development of asthma.

Many studies have focused on asthma risks for single factors such as environmental tobacco smoke (ETS) [4] and nitrogen dioxide $\left(\mathrm{NO}_{2}\right)$ [5], yet multiple exposures are realistically experienced. There is increasing interest in how interactions between air pollutants and allergens promote the development of respiratory disease in childhood [6].

Most of the evidence supporting air pollutants' interaction with allergens has come from in vitro or animal studies $[3,7]$. For example, a study using a mouse model suggested that while ovalbumin inhalation alone was insufficient to trigger the innate immune response, $\mathrm{NO}_{2}$ along with ovalbumin could promote allergic sensitisation and development of asthma [7]. In a rare human study addressing such interactions, a significant association between respiratory symptoms (labelled "bronchitic" but consistent with asthma exacerbation) and particulate matter was only noted in the subset of asthmatic children who owned dogs [8]. In another study, the exposure of mild asthmatics to $\mathrm{NO}_{2}$, at concentrations typical of the home environment, enhanced the drop in airflow associated with inhaled allergen [9]. Because it is possible to at least partially remedy dog allergen (Can-f1) and because it is particularly potent in augmenting pollutant effects [8], we focused on this allergen. The end-points chosen were asthma and bronchial hyperreactivity (BHR), the latter having been littlestudied in this context in spite of its appeal as a quantitative measure of airway reactivity.

\section{AFFILIATIONS}

*Dept of Medicine, University of British Columbia, Vancouver, Canada and

\# Dept of Pediatric Allergy, University of Manitoba, Winnipeg, Canada.

CORRESPONDENCE

C. Carlsten

Vancouver General Hospital 2775 Laurel Street

7th Floor (The Lung Center) Vancouver BC V5Z $1 \mathrm{M} 9$ Canada

E-mail: chris.carlsten@vch.ca

Received:

Nov 242009

Accepted after revision:

May 222010

First published online:

June 072010 
The impact of children's atopic status on the effects of pollutant exposure is unclear. Atopy has often been assumed to be a risk factor for greater respiratory effects associated with air pollution exposures. However, in a recent inner city study of 1,444 children, higher levels of indoor $\mathrm{NO}_{2}$ were most often associated with increased asthma symptoms and decreased peak flows in nonatopic children [10].

Therefore, given that there is significant evidence of the impact of single exposures on airway disease, but that there has been considerably less evaluation of realistic combinations, we hypothesised that early exposure to inhaled gases and particles, in combination with exposure to dog, increases incidence of asthma and BHR in a high-risk birth cohort. We further hypothesised that atopy increases the association between each of these exposures and respiratory end-points. Therefore, the objective of the present study was to evaluate the effect of combined early exposure to Can-f1 plus indoor $\mathrm{NO}_{2}$ or ETS on asthma and BHR in a high-risk birth cohort. We also aimed to assess the impact of atopy on the effects of these exposures. We have previously published on this cohort [11-13] but haven't previously published the results of such analyses considering these pollutants.

\section{METHODS}

\section{Study population}

As described in our previously published study, 545 mothers of infants at high-risk for asthma, from Vancouver and Winnipeg, Canada, were recruited during the third trimester of pregnancy [11]. High-risk was defined as having at least one first-degree relative with asthma or two first-degree relatives with immunoglobulin E-mediated allergic disease. Families were randomised to the control (usual care) or intervention group. The intervention has been previously described in detail; briefly, it was a multifaceted approach to reducing household dust (and related allergens), pets, ETS, and dietary factors thought to promote allergic disease [11].

\section{Exposure assessment prenatally and in year 1}

Most of the exposure assessments and related assays relevant to this birth cohort have been detailed previously [11]; those relevant to the present study are described hereafter. Home visits included questionnaires regarding daycare attendance, dog ownership and the presence of a gas stove before birth and then after birth at 2 weeks, and 4, 8, and 12 months. Cord blood for cotinine (CCot) by radioimmunoassay (Dept of Biochemistry, Brandeis University, Waltham, MA, USA) at birth was quantified in $\mathrm{ng} \cdot \mathrm{mL}^{-1}$ [14]. Dust samples from each household room in which the child spent considerable time were collected at $1 \mathrm{yr}$ after birth from six sites in duplicate, from which dog (Can-f1) allergen levels in $\mu \mathrm{g} \cdot \mathrm{g}^{-1}$ of dust were determined by ELISA with purified monoclonal antibodies (Indoor Biotech, Charlottesville, VA, USA) (lower limit of detection (LLD) of $0.1 \mu \mathrm{g} \cdot \mathrm{g}^{-1}$ [15]). The mean of each duplicate was first determined and then the six means were averaged to yield Can-f1 exposure measure for each child. $\mathrm{NO}_{2}$ was assayed using ion chromatography (Dionex DX-300; Dionex Corporation, Sunnyvale, CA, USA) with suppressed conductivity detection after passive sampling by Palmes tubes in the child's bedroom for 2 weeks. The ratio of urinary cotinine
(UCot):creatinine was assessed at 12 months by radioimmunoassay (Dept of Biochemistry, Brandeis University) [14]. Dog ownership, water leaks and visible mould were defined as positive if they were reported at any point during year 1. Gas stove was defined by its presence at prenatal visit. At the 12-month visit, research personnel performed allergy skin tests with common food and inhalant allergens [12], and the presence of at least three positive $(>3 \mathrm{~mm})$ wheals was diagnostic of atopy.

\section{Outcome assessment at 7 yrs of age}

A single paediatric allergist at each centre was asked to assess subjects for clinical diagnoses of asthma, using a standardised structured questionnaire and definitions that were consistent between centres [11]. BHR was measured [16], and the provocative concentration of methacholine $\left(\mathrm{mg} \cdot \mathrm{mL}^{-1}\right)$ causing a $20 \%$ fall in forced expiratory volume in $1 \mathrm{~s}$ (PC20) was calculated by interpolation of $\mathrm{PC} 20$ values $<8$ and by extrapolation of those $>8$.

\section{Data analysis}

Data points determined to be below the LLD of assay were assigned a value halfway between LLD and zero. Continuous variables were dichotomised at the 50th percentile (median value), with the exception of Can-f1 (dichotomised according to whether or not the levels were $>2 \mu \mathrm{g} \cdot \mathrm{g}^{-1}$ dust) [17] and PC20 (dichotomised according to whether or not the value was $\leqslant 2 \mathrm{mg} \cdot \mathrm{mL}^{-1}$, based on preliminary analysis of maximal sensitivity and specificity for allergist-diagnosed asthma). In a given analysis, pairwise deletion was used for missing data. An OR (95\% CI) for asthma and for BHR was estimated for each individual exposure and for combined exposures, using multiple logistic regression analysis with potential confounders (sex, race, maternal education, history of asthma (in mother, father or siblings), city of residence, atopic status at year 1 , and season); this was entered stepwise (retained if $\mathrm{p}<0.05$ ), while intervention status was forced into the model. Analysis of ORs for asthma and BHR attributable to exposure plus atopy was performed identically. Comparison of ORs, as presented in the results, was performed to assess interactions between exposures and/or atopy at the additive level [18]. We took this approach in anticipation that interactions of the type we were evaluating were more likely to occur on the additive scale; as noted by AнLвом [19] and RothMAN [20], biological interaction typically results in departure from additivity of the independent disease rates. Association between Can-f1 and ownership was assessed using Spearman correlation. The following sensitivity analyses, which were designed, as noted, to address analytical concerns rather than scientific hypotheses, were performed for each model as appropriate: 1) analyses were stratified by intervention status; 2) exposure variables were inputted as continuous (rather than dichotomous); 3) because of the concern that dichotomisation of Can-f1 at $2 \mu \mathrm{g} \cdot \mathrm{g}^{-1}$ dust was not well-established, several alternative cut-offs, including a cut-off at the LLD, were explored; d) dog ownership was substituted for elevated Can-f1; e) for BHR analysis, alternative PC20 cut-off values $\left(3 \mathrm{mg} \cdot \mathrm{mL}^{-1}\right.$ [21] and $4 \mathrm{mg} \cdot \mathrm{mL}^{-1}$ ) were assessed as a further sensitivity analysis since there is no consensus threshold for PC20 in this age group and, alternatively, BHR was analysed as a continuous variable for those children with $\mathrm{PC}_{20} \leqslant 8$ (interpolation of $\mathrm{PC} 20>8$ was considered unreliable). Given the limited sample size for $\mathrm{NO}_{2}$, 
we added water leaks and moulds separately into the multivariate analysis of the primary effect of $\mathrm{NO}_{2}$ to see whether these additional variables would affect the estimate of risk associated with $\mathrm{NO}_{2}$. A priori, we determined that sample size was too limited to allow for analysis of the effect of both combined exposures and atopy (hence, three or more conditions) simultaneously.

\section{RESULTS}

$380(70 \%)$ of the 545 original cohort subjects were re-assessed at 7 yrs of age [12]. Table 1 presents the characteristics of the group as a whole and stratified according to diagnosis. Male sex, having a mother with asthma, and living in Winnipeg were each associated with asthma in univariate analyses; children with asthma were not more likely to be attending daycare facilities. While family history of asthma was common to most subjects, asthma and BHR were present in the minority of subjects. Upon evaluation by the paediatric allergist, asthma was diagnosed in $19 \%$ of the children. 348 children performed technically acceptable methacholine challenge tests, $141(41 \%)$ of whom had BHR. The mean \pm SD PC20 was $3.7 \pm 2.9$. Of those with asthma, $63 \%$ also had BHR; of those without asthma, 35\% also had BHR. Of those with BHR, 29\% were also diagnosed as having asthma; of those without BHR, 12 were diagnosed as having asthma.

The sampling size for each exposure variable is described in table 1 of the supplementary material. Most notably, bedroom $\mathrm{NO}_{2}$ sampling was performed in a majority of the Vancouver

\begin{tabular}{|c|c|c|c|c|c|}
\hline \multirow[t]{2}{*}{ TABLE 1} & \multicolumn{5}{|c|}{$\begin{array}{l}\text { Characteristics of children with and without } \\
\text { asthma and bronchial hyperreactivity (BHR) at } 7 \\
\text { yrs of age }\end{array}$} \\
\hline & & Asthma & No asthma & BHR & No BHR \\
\hline \multicolumn{2}{|l|}{ Subjects $n$} & $71(18.7)$ & 309 (81.3) & $141(40.5)$ & $207(59.5)$ \\
\hline \multicolumn{6}{|l|}{ Race } \\
\hline \multicolumn{2}{|l|}{ White } & 61 (85.9) & $240(77.7)$ & $115(81.6)$ & 159 (76.8) \\
\hline \multicolumn{2}{|l|}{ Non-White } & $10(14.1)$ & 69 (22.3) & $26(18.4)$ & $48(23.2)$ \\
\hline \multicolumn{6}{|l|}{ Sex } \\
\hline \multicolumn{2}{|l|}{ Female } & $24(33.8)$ & $153(49.5)$ & $63(44.7)$ & $98(47.3)$ \\
\hline \multicolumn{2}{|c|}{ Male } & $47(66.2)$ & $156(50.5)$ & 78 (55.3) & 109 (52.7) \\
\hline \multicolumn{6}{|c|}{ History of asthma } \\
\hline \multicolumn{2}{|c|}{ No asthma } & $8(11.3)$ & $77(24.9)$ & $27(19.1)$ & $52(25.1)$ \\
\hline \multicolumn{2}{|c|}{$\begin{array}{l}\text { Father and/or sibling with } \\
\text { asthma }\end{array}$} & $25(35.2)$ & $108(35.0)$ & $52(36.9)$ & $68(32.9)$ \\
\hline \multicolumn{2}{|c|}{ Mother with asthma } & $38(53.5)$ & $124(40.1)$ & $62(44.0)$ & $87(42.0)$ \\
\hline \multicolumn{6}{|c|}{$\begin{array}{l}\text { Maternal post-secondary } \\
\text { education }\end{array}$} \\
\hline \multicolumn{2}{|l|}{ No } & $21(29.6)$ & $64(20.7)$ & $31(22.0)$ & $50(24.2)$ \\
\hline \multicolumn{2}{|l|}{ Yes } & $50(70.4)$ & 245 (79.3) & $110(78.0)$ & $157(75.8)$ \\
\hline \multicolumn{6}{|c|}{ City of residence } \\
\hline \multicolumn{2}{|l|}{ Vancouver } & $23(32.4)$ & $163(52.8)$ & $70(49.6)$ & $105(50.7)$ \\
\hline \multicolumn{2}{|l|}{ Winnipeg } & $48(67.6)$ & $146(47.2)$ & $71(50.4)$ & $102(49.3)$ \\
\hline \multicolumn{6}{|c|}{ Daycare in year 1} \\
\hline \multicolumn{2}{|c|}{ Yes } & $5(7.0)$ & $26(8.4)$ & $12(8.5)$ & $15(7.2)$ \\
\hline \multicolumn{2}{|l|}{ No } & 66 (93.0) & 283 (91.6) & 129 (91.5) & $192(92.8)$ \\
\hline
\end{tabular}

Data are presented as $n(\%)$. subset (155 of 186 homes) but not in any of the Winnipeg homes due to budgetary constraints; mean $\mathrm{NO}_{2}$ was $11.3 \mathrm{ppb}$ (median 10.0 (range 3.4-36.8)). Compared to those without measurements for $\mathrm{NO}_{2}$, those who underwent $\mathrm{NO}_{2}$ sampling were more likely to have a mother with post-secondary education and to be non-White, while no such differences were found in terms of sex and family history. The presence of a gas stove, data for which were available in all children, strongly predicted a significantly higher bedroom $\mathrm{NO}_{2}$ $(\mathrm{p}=0.004)$.

As shown in table 2, with the exception of Can-f1, each single exposure was generally more prevalent amongst asthmatics compared to nonasthmatics; however, following adjusted analysis, these differences were only significant in the case of dog ownership. For BHR, no exposure was significantly more prevalent in those with the condition compared to those without. Atopy was significantly more common in those with asthma and in those with BHR. However, the early exposures were only significantly associated with atopy itself, at either 12 months or $7 \mathrm{yrs}$, in the case of dog ownership and Can-f1 (protective at 12 months and 7 yrs, respectively), as shown in table 3. None of the air pollution exposures levels were significantly different between children in the control group versus those in the intervention group (table 2 of the supplementary material). There was no significant change in risk estimate with the addition of water leaks or visible mould (with data for each of these coming from all 380 children) into the multivariate analyses.

The combination of exposure to Can-f1 and elevated $\mathrm{NO}_{2}$ or elevated CCot conferred an increased risk of asthma relative to having neither such exposure (fig. 1a; also see table 3 of the online supplementary material). In each of the other two exposure combinations, there was a trend towards the combined exposure conferring an increased risk relative to either exposure alone (table 5a of the online supplementary material). For BHR, no combination of exposure conferred an increased risk of asthma relative to having neither such exposure (fig. 1b; see also table 3 of the supplementary material). Figure 2 (and table 4 of the online supplementary material) describes the risk of asthma and BHR if the subject had each of the four increased exposure conditions, atopy or both. Having both atopy and elevated UCot conferred an increased risk for asthma and BHR, compared with having neither risk factor. When comparing the presence of both risk factors to having one risk factor alone (table $5 b$ of the online supplementary material), it is noteworthy that having both atopy and elevated UCot, or both atopy and elevated CCot, confers a greater risk for asthma than having elevated UCot, or elevated CCot, alone (OR 6.6 (95\% CI 2.9-15.1) and OR 5.0 (95\% CI 2.0-12.8), respectively); however, these increased risks were not significantly different from those attributable to atopy alone. In contrast, the risk of BHR with both elevated UCot and atopy is significantly greater than that with atopy alone (OR 3.1 (95\% CI 1.1-8.6)).

Regarding sensitivity analyses (data not shown except where indicated), in summary: stratified analysis revealed patterns of risk within the control group alone, similar to those observed in the cohort as a whole. However, the unstable estimates resulting from this manoeuvre made it difficult to draw 
TABLE 2 Frequency of given exposure amongst those with asthma or bronchial hyperreactivity at 7 yrs of age

\begin{tabular}{|c|c|c|c|c|c|c|}
\hline & Asthma & No asthma & Adjusted $^{\#}$ OR $(95 \% \mathrm{Cl})$ & $\mathrm{PC}_{20}<2$ & $P_{20} \geqslant 2$ & Adjusted $^{\#}$ OR (95\% Cl) \\
\hline \multicolumn{7}{|l|}{ ETS } \\
\hline UCot $>$ 50th percentile & $43 / 71(60.6)$ & 149/299 (49.8) & $1.3(0.7-2.4)$ & 71/136 (52.2) & 102/202 (50.5) & $1.1(0.7-1.8)$ \\
\hline Gas stove & 8/71 (11.3) & 29/309 (9.4) & $1.4(0.6-3.6)$ & 15/141 (10.6) & 19/207 (9.2) & $1.2(0.6-2.5)$ \\
\hline \multicolumn{7}{|l|}{$\mathrm{NO}_{2}$} \\
\hline$\geqslant 2 \mu \mathrm{g} \cdot \mathrm{g}^{-1}$ dust & $30 / 71(42.3)$ & 102/308 (33.1) & $1.3(0.7-2.4)$ & 41/141 (29.1) & 77/206 (37.4) & $0.7(0.4-1.1)$ \\
\hline Dog ownership & $18 / 70(25.7)$ & 42/307 (13.7) & $2.7(1.3-5.6)^{\star}$ & 20/138 (14.5) & $34 / 207(16.4)$ & $1.0(0.5-1.8)$ \\
\hline Atopy & $34 / 71$ (47.9) & 56/305 (18.4) & $5.5(3.0-10.2)^{*}$ & $47 / 140(33.6)^{*}$ & 35/204 (17.2) & $2.4(1.5-4.0)^{*}$ \\
\hline
\end{tabular}

Data are presented as $\mathrm{n} / \mathrm{N}(\%)$, unless otherwise stated. The denominator varies within a given cell and in given row because it represents only those within the column for whom corresponding exposure data also exist. PC20: provocative concentration of methacholine causing a $20 \%$ fall in forced expiratory volume in $1 \mathrm{~s}$; ETS: environmental tobacco smoke; CCot: cord blood for cotinine; UCot: urinary cotinine; $\mathrm{NO}_{2}$ : nitrogen dioxide. ${ }^{\#}$ : each model is adjusted for group allocation (intervention) plus any retained baseline characteristic (race, sex, history of asthma, maternal education, city of residence, season, child's atopic status (not an additional covariate in models with atopy already in model as the predictor variable)). *: $p<0.05$.

reasonable conclusions. Models with continuous exposure variables were complicated by clustering of data for Can-f1 levels at the lower end of the data range and an inability to transform such data into an appropriate distribution for analysis. There was no significant change to the results by either: using cut-off thresholds other than $2 \mu \mathrm{g} \cdot \mathrm{g}^{-1}$ for elevated Can-f1 dust (including a cut-off at LLD) or $2 \mathrm{mg} \cdot \mathrm{mL}^{-1}$ methacholine for PC20; nor by analysing BHR as a continuous variable (for 268 children with PC20 $\leqslant 8$ ). Regarding substitution of $\operatorname{dog}$ ownership for allergen, exposure to dog allergen $\geqslant 2 \mu \mathrm{g} \cdot \mathrm{g}^{-1}$ dust was significantly correlated with dog ownership $(r=0.53 ; p=0.01)$; ORs were generally consistent when dog ownership was substituted for Can-f1 in regression analyses. However, an exception was that, in contrast to Can-f1, dog ownership alone significantly elevated risk of asthma on multivariate analysis (table 2), and dog ownership combined with elevated UCot conferred an increased risk of asthma (OR 5.8 (95\% CI 2.1-15.7)).

\section{DISCUSSION}

In this high-risk birth cohort, combined early exposure to dog (elevated Can-f1 levels or dog ownership) plus elevated $\mathrm{NO}_{2}$ or ETS conferred an increased risk of incident asthma. Although this was more apparent when comparing children with both exposures to children with neither exposure (fig. 1; see also table 3 of the online supplementary material) than when comparing those with both exposures to those with either exposure (table 5 of the online supplementary material), the data as a whole suggest synergy on the additive level [19]. Our findings are supported by related literature on the adjuvant effects between gaseous and particulate exposures and allergens [7, 22-25], particularly in the in utero context,

TABLE 3 Frequency of given exposure amongst those with atopy at 12 months and at 7 yrs of age

\begin{tabular}{|c|c|c|c|c|c|c|}
\hline & Atopy at 12 months & $\begin{array}{l}\text { No atopy at } \\
12 \text { months }\end{array}$ & $\begin{array}{l}\text { Adjusted }{ }^{\#} \\
\text { OR }(95 \% \mathrm{Cl})\end{array}$ & $\begin{array}{c}\text { Atopy at } \\
7 \mathrm{yrs} \text { of age }\end{array}$ & $\begin{array}{l}\text { No atopy } \\
\text { at } 7 \text { yrs of age }\end{array}$ & $\begin{array}{l}\text { Adjusted }^{\#} \\
\text { OR (95\% Cl) }\end{array}$ \\
\hline \multicolumn{7}{|l|}{ ETS } \\
\hline CCot $>50$ th percentile & 35/63 (55.6) & 102/213 (47.9) & $1.4(0.8-2.5)$ & 71/123 (57.7) & $61 / 145(42.1)$ & $1.4(0.8-2.5)$ \\
\hline UCot $>50$ th percentile & $41 / 88(46.6)$ & 149/278 (53.6) & $0.8(0.5-1.3)$ & 89/166 (53.6) & 97/194 (50.0) & $0.9(0.6-1.5)$ \\
\hline Gas stove & 10/90 (11.1) & 26/286 (9.1) & $1.3(0.6-2.8)$ & 15/167 (9.0) & 22/200 (11.0) & $0.9(0.4-1.9)$ \\
\hline \multicolumn{7}{|l|}{ Can-f1 } \\
\hline$\geqslant 2 \mu \mathrm{g} \cdot \mathrm{g}^{-1} \mathrm{dust}$ & 28/89 (31.5) & 103/286 (36.0) & $0.9(0.5-1.5)$ & 49/166 (29.5) & 78/200 (39.0) & $0.6(0.4-0.9)^{*}$ \\
\hline Dog ownership & $6 / 88(6.8)$ & $54 / 285(18.9)$ & $0.3(0.1-0.8)^{*}$ & 23/165 (13.8) & 34/199 (17.0) & $0.6(0.3-1.2)$ \\
\hline
\end{tabular}



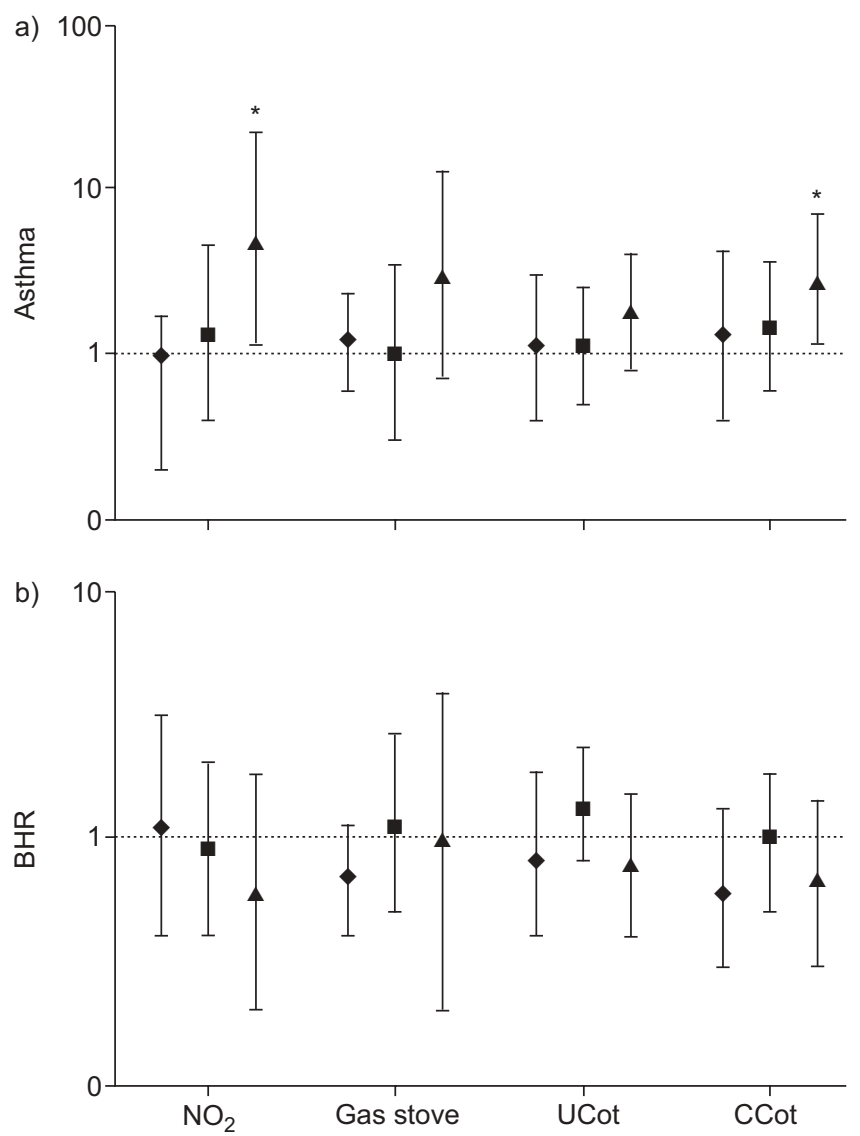

FIGURE 1. Risk of a) asthma and b) bronchial hyperreactivity (BHR) for exposure to dog allergen (Can-f1) only $(\bullet)$, other exposure only $(\mathbf{\square})$, or combined (dog plus other given) exposures ( $(\mathbf{\Lambda})$. Data points and whiskers represent OR $(95 \%$ $\mathrm{Cl}$ ). $\mathrm{NO}_{2}$ : nitrogen dioxide; UCot: urinary cotinine; Ccot: cord blood for cotinine. *: $p<0.05$. Cls and variables withstanding stepwise regression processes are presented in table 1 of the online supplementary material. Exposures were defined as per the Methods section. Within each set of three ORs, the sample size contributing to each OR was identical and defined by the available sampling size of the given pollutant for a given set of three ORs (i.e. $\mathrm{NO}_{2}=155$, gas stove $=379$, $U \mathrm{Cot}=370, \mathrm{CCot}=279$ ). No "intercondition" comparisons were significant, as further detailed in table $5 \mathrm{a}$ of the online supplementary material.

where ETS exposure can lead to a T-helper cell type 2 cytokine bias [26]. Given that exposure did not lead to atopy itself at either 12 months or 7 yrs, and may even have been protective in the case of dog, it appears that observed effects of combined exposure on asthma are not simply secondary to exposurerelated atopy.

The second notable finding of the present study was that subjects with several non-allergen exposures in the presence of atopy were at increased risk of asthma and BHR. The magnitude of these effects was strikingly higher than that noted with combined dog and non-allergen exposures summarised above. While atopy was generally a stronger independent risk factor for these outcomes than non-allergen exposures alone, non-allergen exposures (particularly ETS) appeared to augment the risk due to atopy for these outcomes; there was a pattern for such effects beyond that anticipated on a purely additive basis. This was most clearly seen in the
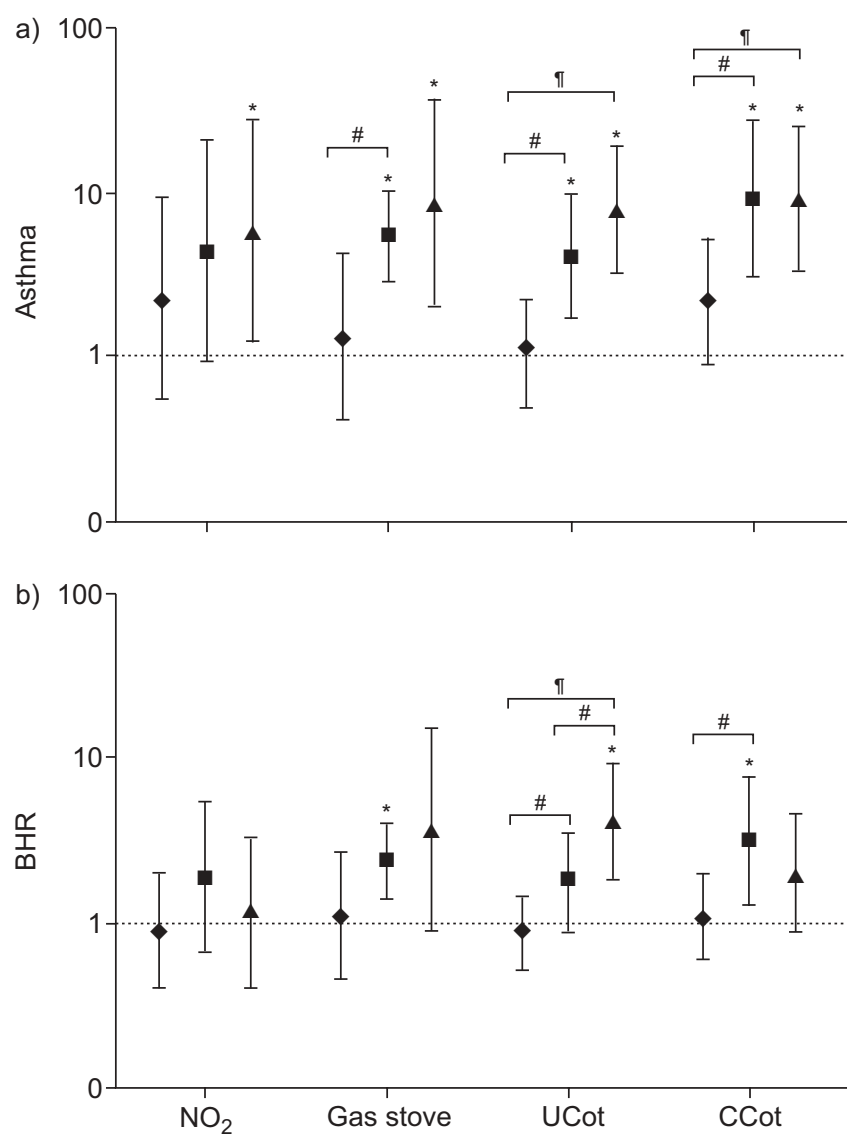

FIGURE 2. Risk of a) asthma and b) bronchial hyperreactivity (BHR) for given exposure only ( ), atopy only ( $\mathbf{\square})$ or combined (atopy plus other given) exposures (A). Data points and whiskers represent OR ( $95 \% \mathrm{Cl}$ ). $\mathrm{NO}_{2}$ : nitrogen dioxide; UCot: urinary cotinine; Ccot: cord blood for cotinine. *: $p<0.05$; ${ }^{*}$ : $p<0.05$ for comparison of conditions to each other (as further detailed in table $5 \mathrm{~b}$ of the online supplementary material); ${ }^{\bullet}: p<0.005$ for comparison of conditions to each other (as further detailed in table $5 \mathrm{~b}$ of the online supplementary material). Cls and variables withstanding stepwise regression process are presented table 2 of the online supplementary material. Exposures were defined as per the Methods section. Within each set of three ORs, the sample size contributing to each OR was identical and defined by the available sampling size of the given pollutant for a given set of three ORs (i.e. $\mathrm{NO}_{2}=155$, gas stove=379, UCot=370, CCot=279).

increased risk of BHR associated with elevated UCot exposure in atopic subjects. Although the importance of a child's atopic status in modulating the effect of ETS on incident airways disease has been inferred by examination in prior studies [4], direct demonstration of the atopic modulation of ETS's effect on incident BHR is novel and noteworthy, particularly given our unique in utero data. Interestingly, the finding of increased risk for asthma in those with elevated $\mathrm{NO}_{2}$ exposure and atopy is at odds with the findings of others [10], who noted that it was in children without atopy that $\mathrm{NO}_{2}$ posed more risk. This is perhaps due to the high-risk nature of our cohort and/or the assessment of exposure at an earlier age associated with particular vulnerability to the development of asthma. Other features of our study, distinct from that of KATTAN et al. [10], were the longitudinal design and the inclusion of nonasthmatics within the analysis. 
There is biological plausibility to our findings; while the precise mechanisms are poorly understood, evidence to date points to gaseous- and particle-driven damage of epithelial cell membranes that allows for augmentation of inflammatory pathways [15] and related immunological phenomena [7, 27]. The consistent patterns we observed for a combined effect of ETS exposure (as measured by cotinine in both cord blood and urine) and atopy is supported by limited prior investigation [28] and motivates further mechanistic studies.

There are limitations to our study. First, the sample size limited our ability to more precisely assess the magnitude of combined exposure effects. In particular, indoor $\mathrm{NO}_{2}$ levels were only available in a subset of the cohort. Because indoor $\mathrm{NO}_{2}$ levels were not available for Winnipeg homes, this is a potential source of bias to the extent that unmeasured confounders associated with $\mathrm{NO}_{2}$ in Vancouver may have been present but unaccounted for. For example, $\mathrm{NO}_{2}$ may be associated with bedroom ventilation or infiltration characteristics, for which we have no data. It is somewhat reassuring that the analyses using gas stoves (a proxy for indoor $\mathrm{NO}_{2}$ but available for the entire cohort) led to similar results and that the presence of water leaks or mould did not change the risk estimates; however, validation of our findings with a larger sample size and more data on home characteristics is desirable. A larger sample size would also help to better delineate the difference in risk between single and dual exposures in such contexts; although our analyses overall demonstrate patterns consistent with additive-level synergy, there are several exposure combinations for which limited power makes it difficult to clearly differentiate between the effects of single versus dual exposures. Secondly, the study lacks subclinical indicators (i.e. airway cell types and markers of inflammation or airway remodelling) allowing better elucidation of mechanisms relevant to our findings; this suggests a direction for future research. Thirdly, the study was originally designed with an intervention and we know that the intervention was effective in resolving some of the risk factors of concern in these children; therefore, this is not the ideal context for a study of exposure interactions. However, the exposure to elevated air pollutant levels was not significantly different between control and intervention groups (table 2 of the online supplementary material). Fourthly, it might not be possible to generalise the results to a non-high risk cohort; however, even populationbased scenarios include high-risk individuals who may be at risk in a similar way to that demonstrated in our risk-enriched cohort. Finally, we did not have data on endotoxin exposure in early life. We have recently documented that dog ownership alone in early life is associated with increased risk for asthma [13]; because ownership was not associated with increased sensitisation to Can-f1 [29], this raises the possibility that endotoxin dominates the effect of dog ownership, as suggested by others [8]. That the results of the present study were similar whether "dog" was represented by allergen or ownership supports the need for further research to discriminate between allergen and endotoxin effects.

Despites these limitations, there are several major strengths and novel features notable in our study. First, while the overall cohort size was modest, the number of asthma cases (by virtue of the cohort's high-risk nature) was considerable; we were able to analyse more cases at 7 yrs of age than the hallmark
German Multicentre Asthma Study [30]. Secondly, the birth cohort was very well characterised in terms of family characteristics and environmental exposures, not only at several intervals after birth but also in the peri-partum period. In particular, the data reflecting in utero ETS exposure in conjunction with the collective study data are unique; that ETS was quantified by cotinine, rather than using responses to questionnaires vulnerable to recall bias, is another noteworthy strength of this study and may account for our ability to detect significant risk with a modest sample size. Furthermore, obtaining methacholine challenge data, very rarely achievable in this age group, along with well-standardised diagnoses from paediatric allergists, further tempers the concern of modest sample size and makes this study particularly valuable in terms of phenotypic precision.

To summarise, in children at high risk for asthma, co-exposure to dog along with gaseous and particulate pollutants appears to increase risk for asthma. Atopic children appear to be at increased risk for both asthma and BHR when exposed to nonallergen insults, particularly ETS exposure, as represented by the metabolite cotinine.

\section{SUPPORT STATEMENT}

The present study was supported by the Canadian Institutes of Health Research, the BC Lung Association and the Manitoba Medical Service Foundation.

\section{STATEMENT OF INTEREST}

None declared.

\section{ACKNOWLEDGEMENTS}

We thank the cohort children and their families for their participation and P. Brasher (University of British Columbia, Vanconver, Canada), D. Dockery (Harvard University, Cambridge, MA, USA), S. Greenland (UCLA School of Public Health, Los Angeles, CA, USA), and R. Hosseini (University of British Columbia, Vanconver, Canada) for helpful review and input regarding study methodology.

\section{REFERENCES}

1 Global Initiative for Asthma Guidelines. www.ginasthma.com/ Reports.asp?11=2\&12=1 Date last accessed: November 25, 2010.

2 Sporik R, Platts-Mills TA. Allergen exposure and the development of asthma. Thorax 2001; 56: Suppl. 2, ii58-ii63.

3 Pearce N, Douwes J, Beasley R. Is allergen exposure the major primary cause of asthma? Thorax 2000; 55: 424-431.

4 Vork KL, Broadwin RL, Blaisdell RJ. Developing asthma in childhood from exposure to secondhand tobacco smoke: insights from a meta-regression. Environ Health Perspect 2007; 115: 1394-1400.

5 van Strein RT, Gent JF, Belanger K, et al. Exposure to NO2 and nitrous acid and respiratory symptoms in the first year of life. Epidemiology 2004; 15: 471-478.

6 Traidl-Hoffmann C, Jakob T, Behrendt H. Determinants of allergenicity. J Allergy Clin Immunol 2009; 123: 558-566.

7 Bevelander M, Mayette J, Whittaker LA, et al. Nitrogen dioxide promotes allergic sensitization to inhaled antigen. J Immunology 2007; 179: 3680-3688.

8 McConnell R, Berhane K, Molitor J, et al. Dog ownership enhances symptomatic responses to air pollution in children with asthma. Environ Health Perspect 2006; 114: 1910-1915. 
9 Tunnicliffe WS, Burge PS, Ayres JG. Effect of domestic concentrations of nitrogen dioxide on airway responses to inhaled allergen in asthmatic patients. Lancet 1994; 334: 1733-1736.

10 Kattan M, Gergen PJ, Eggleston P, et al. Health effects of indoor nitrogen dioxide and passive smoking on urban asthmatic children. J Allergy Clin Immunol 2007; 120: 618-624.

11 Chan-Yeung M, Manfreda J, Dimich-Ward H, et al. A randomized controlled study on the effectiveness of a multifaceted intervention program in the primary prevention of asthma in high-risk infants. Arch Pediatr Adol Med 2000; 154: 657-663.

12 Chan-Yeung M, Ferguson A, Watson W, et al. The Canadian Childhood Asthma Primary Prevention Study: outcomes at 7 years of age. J Allergy Clin Immunol 2005; 116: 49-55.

13 Chan-Yeung M, Hegele RG, Dimich-Ward $H$, et al. Early environmental determinants of asthma risk in a high-risk birth cohort. Pediatric Allergy Immunology 2008; 19: 482-489.

14 Becker AB, Manfreda J, Ferguson AC, et al. Breast-feeding and environmental tobacco smoke exposure. Arch Pediatr Adolesc Med 1999; 153: 689-691.

15 Barck C, Lundahl J, Halldén G, et al. Brief exposures to NO2 augment the allergic inflammation in asthmatics. Environ Res 2005; 97: 56-66.

16 Cockcroft DW, Killian DN, Mellon JJ, et al. Bronchial reactivity to inhaled histamine: a method and clinical survey. Clin Allergy 1977; 7: 235-243.

17 Gent JF, Belanger K, Triche EW, et al. Association of pediatric asthma severity with exposure to common household dust allergens. Environ Res 2009; 109: 768-774.

18 Greenland S. Basic problems in interaction assessment. Environ Health Perspect 1993; 101: 59-66.

19 Ahlbom A, Alfredsson L. Interaction: a word with two meanings creates confusion. Eur J Epidemiol 2005; 20: 563-564.

20 Rothman KJ. Epidemiology: An Introduction. NewYork, Oxford University Press, 2002.
21 Godfrey S. Bronchial hyper-responsiveness in children. Paediatr Respir Rev 2000; 1: 148-155.

22 Diaz-Sanchez D, Dotson AR, Takenaka H, et al. Diesel exhaust particles induce local IgE production in vivo and alter the pattern of IgE messenger RNA isoforms. J Clin Invest 1994; 94: 1417-1425.

23 Diaz-Sanchez D, Tsien A, Fleming J, et al. Combined diesel exhaust particulate and ragweed allergen challenge markedly enhances human in vivo nasal ragweed-specific $\operatorname{IgE}$ and skews cytokine production to a T helper cell 2-type pattern. J Immunol 1997; 158: 2406-2413.

24 Fujieda S, Diaz-Sanchez D, Saxon A. Combined nasal challenge with diesel exhaust particles and allergen induces in vivo IgE isotype switching. Am J Respir Cell Mol Biol 1998; 19: 507-512.

25 Hashimoto K, Ishii Y, Uchida Y, et al. Exposure to diesel exhaust exacerbates allergen-induced airway responses in guinea pigs. Am J Respir Crit Care Med 2001; 164: 1957-1963.

$26 \mathrm{Yu} \mathrm{M}$, Zheng $\mathrm{X}$, Peake J, et al. Perinatal environmental tobacco smoke exposure alters the immune response and airway innervation in infant primates. J Allergy Clin Immunol 2008; 122: 640-647.

27 Penn AL, Rouse RL, Horohov DW, et al. In utero exposure to environmental tobacco smoke potentiates adult respones to allergen in BALB/c mice. Environ Health Perspect 2007; 115: 548-555.

28 Martinez FD, Antognoni G, Marci F, et al. Parental smoking enhances bronchial responsiveness in nine-year old children. Am Rev Respir Dis 1988; 138: 518-523.

29 Chan-Yeung $M$, Becker $A B$, Ferguson $A$, et al. Indoor allergen exposure, sensitization and development of asthma in a high risk cohort. Am J Respir Crit Care Med 2008; 177: A186.

30 Lau S, Illi S, Sommerfeld C, et al. Early exposure to house-dust mite and cat allergens and development of childhood asthma: a cohort study. Multicentre Allergy Study Group. Lancet 2000; 356: 1392-1397. 